\title{
ICCM 2013 in Taipei
}

\section{by Jing Yu \\ Department of Mathematics, National Taiwan University, Taipei}

The 6th ICCM will be held from July 14 to 19, 2013 in Taipei. You are cordially invited to join this great event! The Sixth International Congress of Chinese Mathematicians (ICCM) July 2013 in Taipei.

- Start: 09:00, July 14, 2013

- End: 18:00, July 19, 2013

- Opening ceremony: July 14, in the Big Hall of the Grand Hotel, Taipei.

- Lectures and invited talks: July 15 to July 19, on the campus (Core subjects Classroom Building, Liberal Education Classroom Building) of National Taiwan University, Taipei.

ICCM is a triennial event that brings together Chinese and overseas mathematicians to discuss the latest research developments in pure, applied mathematics and Statistics.

During the opening of the ICCM 2013, you will witness the presentation of the Morningside Medals, the New World Mathematics Awards, and other awards. The Morningside medal is the most prestigious award for young Chinese mathematicians. During the conference, there will be thirty plenary talks, several Morningside talks by international speakers, and over one hundred sixty 45-minute talks. The talks cover a full range of subjects in the mathematical sciences, from number theory, geometry, and differential equations to statistics and bio-mathematics. As in the past ICCM, we are also inviting a number of great mathematicians from the world to give morningside lectures in mathematics. Among the distinguished guests who have agreed to join us in 2013, there is the renowned mathematician Jean-Pierre Serre from France, who will deliver a special lecture-the title of the lecture is "The genesis of etale cohomology (from the German school in the 30's to Deligne's proof of Weil's conjecture)." Also confirmed are J. Coates from Cambridge University, S. Osher from UCLA, D. Goldfeld from Columbia, and B. Engquist from University of Texas at Austin. There is also a public lecture on Juggling Mathematics and Magic in the opening day of ICCM, to be delivered by R. Graham (UC San Diego, see the short article written by him is in this Notice).
The first Congress was held in December 1998 in Beijing. The second Congress took place in Taipei 2001, then in Hong Kong, in Hangzhou, and Beijing again in 2010. After twelve years, the ICCM 2013 will return to Taipei. We expect there to be more than one thousand participants and more than two hundred presentations covering a broad spectrum of mathematical sciences.

We expect that there to be more than one thousand participants of 2013 ICCM. We have tried to allocate a limited amount of grant to support young people to ICCM, particularly those young Chinese mathematicians from less developed countries, the deadline for application is end of December 2012. For all the invited speakers and the coming committee members, we booked already the Howard hotel and Just Sleep @ NTU on campus, and K Hotel, Taipei Dunnan near Campus. For the other participants, we are contracting several hotels in Taipei with information on the ICCM website provided before the Chinese new year. We listed the necessary documents for applying for the Exit and Entry permit on the ICCM website. We sent out a note to people who are in China at the beginning of February to remind them to register online and turn in the documents for the permit by February 28, 2013. We will help applying for the visa for people around the end of April and once we get the permits from the government, we will mail them out to the participants. We will arrange for buses to pick up the participations from Taoyuan International airport and bring them to the main Hotels and National Taiwan University on $7 / 13$. The bus will go out every two hours. We will announce the schedule of buses and information on the ICCM 2013 website. All secretaries of TIMS, NCTS (Hsinchu, Taipei) joined the administrative group, with also helps from secretaries of math dept (NTU) and Inst. Math (Academia Sinica). For up-to-dating information on ICCM 2013, please visit its website: http://iccm.tims.ntu.edu.tw.

\section{Tentative Schedule}

July 14, 2013

09:00-09:40 Opening Address and Welcoming Remarks 
09:40-12:00 Presentation of Morningside Medals of

Mathematics, also International Cooperation award and Chern Prize

12:45-13:30 Press Conference

14:00-15:15 Symposium: Panel discussion

The following are confirmed panel members, prominent scholars from mainland are being invited.

\section{Title: Government Policy on Fundamental Science}

Panelists:

Cyrus C.Y. Chu (Minister of National Science Council)

Way Kuo (President of City University of Hong Kong)

Jin-Pyng Wang (President of the Legislative Yuan)

Chi-Huey Wong (President of Academia Sinica)

15:30-16:15 Symposium: Public lecture

Title: The Art of Bridge Building

Speaker: C. L. Liu

16:15-17:00 Symposium: Public lecture

Title: Juggling Mathematics and Magic

Speaker: Ron Graham (University of California, San Diego) 18:00-21:00 New World Mathematics Awards Evening

The honorary chair of ICCM is Professor Shing-Tung Yau from Harvard University. The following lists are the committees of the 6th ICCM.

\section{Scientific Committee}

Ching-Li Chai (Institute of Mathematics, Academia Sinica/ University of Pennsylvania), Shiu-Yuen Cheng (The Hong Kong University of Science and Technology), Jiaxing Hong (Fudan University), Thomas Yizhao Hou (California Institute of Technology), Tze Leung Lai (Stanford University), Wen-Ching Winnie Li (National Center for Theoretical Sciences/ National Tsing Hua University), Ker-Chau Li (Academia Sinica), Jun Li (Stanford University), Chang-Shou Lin (National Taiwan University), Kefeng Liu (Zhejiang University/ University of California, Los Angeles), Chi-Wang Shu (Brown University), Xiaotao Sun (Academy of Mathematics and Systems Science, Chinese Academy of Sciences), Mu-Tao Wang (Columbia University), Wing Hung Wong (Stanford University), Nanhua Xi (Academy of Mathematics and Systems Science, Chinese Academy of Sciences), Jie Xiao (Tsinghua University), Zhou Ping Xin (The Chinese University of Hong Kong), Lo Yang (Academy of Mathematics and Systems Science, Chinese Academy of Sciences), Shing-Tung Yau (Harvard University), Horng-Tzer Yau (Harvard University), Jing Yu (National Taiwan University), Ya-xiang Yuan (Academy of Mathematics and Systems Science, Chinese Academy of Sciences), Shou-Wu Zhang (Princeton University), Xi-Ping Zhu (Sun Yat-Sen University)

\section{Executive Committee}

Tony F. Chan (Hong Kong University of Science and Technology), Gerard Jennhwa Chang (National Taiwan University), Louis H. Y. Chen (National University of Singapore), Chongqing Cheng (Nanjing University), Fan Chung Graham (University of California, San Diego), Boling Guo (Institute of Applied Physics and Computational Mathematics), Lei Guo (Academy of Mathematics and Systems Science, Chinese Academy of Sciences), Hesheng $\mathrm{Hu}$ (Fudan University), Sen $\mathrm{Hu}$ (University of Science and Technology of China), Ka-Sing Lau (The Chinese University of Hong Kong), Banghe Li (Academy of Mathematics and Systems Science, Chinese Academy of Sciences), Tatsien Li (Fudan University), Song-Sun Lin (National Chiao Tung University), Tai-Ping Liu (Academia Sinica), Yiming Long (Nankai University), Zhi-Ming Ma (Academy of Mathematics and Systems Science, Chinese Academy of Sciences), Ngaiming Mok (The University of Hong Kong), Yum-Tong Siu (Harvard University), Chuu-Lian Terng (University of California, Irvine), Chien-Fu Jeff Wu (Georgia Institute of Technology), Jyh-Yang Wu (National Chung Cheng University), Jie Xiao (Tsinghua University)

\section{Local Committee}

Ching-Li Chai (Institute of Mathematics, Academia Sinica/ University of Pennsylvania), Shun-Cheng Chang (National Taiwan University), Jung-Kai Chen (National Center for Theoretical Sciences), Kuo-Chang Chen (National Tsing Hua University), Shun-Jen Cheng (Institute of Mathematics, Academia Sinica), Liang-Chung Hsia (National Taiwan Normal University), Ming-Chih Lai (National Chiao Tung University), Ching Hung Lam (National Center for Theoretical Sciences), Yng-Ing Lee (National Taiwan University), Wen-Ching Winnie Li (National Center for Theoretical Sciences/ National Tsing Hua University), Ko-Wei Lih (Academia Sinica), Chang-Shou Lin (National Taiwan University), Tai-Chia Lin (National Taiwan University), Shuenn-Jyi Sheu (National Central University), Chin-Lung Wang (Taida Institute for Mathematical Sciences), Jenn-Nan Wang (National Taiwan University), Tzu-Yueh Julie Wang (Mathematics Research Promotion Center), Chia-Fu Yu (Academia Sinica), Jing Yu (Taida Institute for Mathematical Sciences)

\section{Plenary Speakers}

The scientific committee has already invited the following thirty ICCM Plenary Speakers:

\begin{tabular}{ll}
\hline Ching-Li Chai & $\begin{array}{l}\text { Institute of Mathematics, } \\
\text { Academia Sinica, University of } \\
\text { Pennsylvania }\end{array}$ \\
\hline Bing-Long Chen & Sun Yat-Sen University \\
\hline
\end{tabular}




\begin{tabular}{|c|c|}
\hline Gui-Qiang G. Chen & University of Oxford \\
\hline Hanfu Chen & $\begin{array}{l}\text { Academy of Mathematics and } \\
\text { Systems Science, Chinese } \\
\text { Academy of Sciences }\end{array}$ \\
\hline Ching-Shui Cheng & $\begin{array}{l}\text { University of California, } \\
\text { Berkeley }\end{array}$ \\
\hline Shun-Jen Cheng & $\begin{array}{l}\text { Institute of Mathematics, } \\
\text { Academia Sinica }\end{array}$ \\
\hline Guizhen Cui & $\begin{array}{l}\text { Academy of Mathematics and } \\
\text { Systems Science, Chinese } \\
\text { Academy of Sciences }\end{array}$ \\
\hline Fuquan Fang & Capital Normal University \\
\hline Baohua Fu & $\begin{array}{l}\text { Academy of Mathematics and } \\
\text { Systems Science, Chinese } \\
\text { Academy of Sciences }\end{array}$ \\
\hline Wee Teck Gan & $\begin{array}{l}\text { National University of } \\
\text { Singapore }\end{array}$ \\
\hline Yan Guo & Brown University \\
\hline Thomas Yizhao Hou & $\begin{array}{l}\text { California Institute of } \\
\text { Technology }\end{array}$ \\
\hline Yi-Jen Lee & $\begin{array}{l}\text { The Chinese University of } \\
\text { Hong Kong }\end{array}$ \\
\hline Haizhong Li & Tsinghua University \\
\hline Bong H. Lian & Brandeis University \\
\hline Wen-Wei Lin & $\begin{array}{l}\text { National Chiao Tung } \\
\text { University }\end{array}$ \\
\hline Jun Liu & Harvard University \\
\hline Chi-Wang Shu & Brown University \\
\hline Luen Fai Tam & $\begin{array}{l}\text { The Chinese University of } \\
\text { Hong Kong }\end{array}$ \\
\hline Ye Tian & $\begin{array}{l}\text { Morningside Center of Math- } \\
\text { ematics, Chinese Academy of } \\
\text { Sciences; Academy of Math- } \\
\text { ematics and Systems Science, } \\
\text { Chinese Academy of Sciences }\end{array}$ \\
\hline Tai-Peng Tsai & University of British Columbia \\
\hline Yu-Jong Tzeng & Harvard University \\
\hline Chin-Lung Wang & $\begin{array}{l}\text { Taida Institute for } \\
\text { Mathematical Sciences }\end{array}$ \\
\hline Xu-Jia Wang & Australian National University \\
\hline Jie Xiao & Tsinghua University \\
\hline Horng-Tzer Yau & Harvard University \\
\hline Stephen Yau & Tsinghua University \\
\hline Zhiwei Yun & Stanford University \\
\hline
\end{tabular}

\begin{tabular}{ll}
\hline Wei Zhang & Columbia University \\
\hline Xinwen Zhu & Northwestern University \\
\hline
\end{tabular}

For the 45 minute Invited Speakers, we invited 180 experts in all fields, which is divided into 10 sections:

1. Number Theory, Automorphic Forms and Arithmetic Geometry, 2. Algebra, History of Mathematics, 3. Discrete Mathematics, Combinatorics, 4. Algebraic Geometry, Complex Geometry, 5. Geometric Analysis, Differential Geometry, Symplectic Geometry, 6. Topology, Geometry and Mathematical Physics, 7. Statistical Theory, Methods, Applications, Probability, Financial Mathematics, 8. Dynamical System, Control Theory, Optimization, Functional Analysis, Fractals, 9. Computational Mathematics, Imaging, and Mathematical Biology, 10. Partial Differential Equation.

\section{Number Theory, Automorphic Forms and Arithmetic Geometry}

Chieh-Yu Chang (National Tsing Hua University), Mei-Chu Chang (University of California, Riverside), Lei Fu (Nankai University), Shaofang Hong (Sichuan University), Ming-Lun Hsieh (National Taiwan University), Dihua Jiang (University of Minnesota), Kai-Wen Lan (University of Minnesota), Hongze Li (Shanghai Jiao Tong University), Wen-Ching Winnie Li (National Center for Theoretical Sciences), Wen-Wei Li (Morningside Center of Mathematics, Chinese Academy of Sciences), Jianya Liu (Shandong University), Sheng-Chi Liu (Texas A \& M University), Yifeng Liu (Massachusetts Institute of Technology), Ling Long (Iowa State University), Guangshi Lu (Shandong University), Binyong Sun (Academy of Mathematics and Systems Science, Chinese Academy of Sciences), Yichao Tian (Academy of Mathematics and Systems Science, Chinese Academy of Sciences), Jilong Tong (Université de Bordeaux 1), Dasheng Wei (Academy of Mathematics and Systems Science, Chinese Academy of Sciences), Fei Xu (Capital Normal University), Tonghai Yang (University of Wisconsin-Madison), Yi-Fan Yang (National Chiao Tung University), Chia-Fu Yu (Institute of Mathematics, Academia Sinica), Jiu-Kang Yu (The Chinese University of Hong Kong), Lei Zhang (Boston College), Weizhe Zheng (Morningside Center of Mathematics, Chinese Academy of Sciences), Chengbo Zhu (National University of Singapore), Yongchang Zhu (The Hong Kong University of Science and Technology)

\section{Algebra, History of Mathematics}

Bangming Deng (Beijing Normal University), Xuhua He (The Hong Kong University of Science and Technology), Jun $\mathrm{Hu}$ (Beijing Institute of Technology), Ming-Chang Kang (National Taiwan University), Ching Hung Lam (Institute of Mathematics, Academia Sinica), Ngau Lam (National Cheng Kung University), Wenlin Li (Academy of Mathematics and Systems Science, Chinese Academy of 
Sciences), Anjing Qu (Northwest University, China), Peng Shan (Massachusetts Institute of Technology/ CNRS, Université de Caen), Man Keung Siu (The University of Hong Kong), Jiping Zhang (Peking University)

\section{Discrete Mathematics, Combinatorics}

Gerard Jennhwa Chang (National Taiwan University), Fan Chung Graham (University of California, San Diego), Thomas Lam (University of Michigan), Yusheng Li (Tongji University), Linyuan Lu (University of South Carolina), Xingxing Yu (Georgia Institute of Technology), Cun-Quan Zhang (West Virginia University), Xiao-Dong Zhang (Shanghai Jiao Tong University), Xuding Zhu (Zhejiang Normal University)

\section{Algebraic Geometry, Complex Geometry}

Jung-Kai Chen (National Taiwan University), Qile Chen (Columbia University), Chen-Yu Chi (National Taiwan University), Quo-Shin Chi (Washington University in St. Louis), Rong Du (East China Normal University), Yi Hu (University of Arizona), Xiaojun Huang (Rutgers University), Hui-Wen Lin (National Taiwan University), Steven Lu (Université du Québec à Montréal), Yat Sun Poon (University of California, Riverside), Jian Song (Rutgers University), Shenghao Sun (Mathematical Sciences Center , Tsinghua University), Song Sun (Imperial College London), Hsian-Hua Tseng (Ohio State University), Bun Wong (University of California, Riverside), Qihong Xie (Fudan University), Jeng-Daw Yu (National Taiwan University), De-Qi Zhang (National University of Singapore), Yi Zhang (Fudan University), Fangyang Zheng (Ohio State University)

\section{Geometric Analysis, Differential Geometry, Symplectic Geometry}

Huai-Dong Cao (Lehigh University), Shu-Cheng Chang (National Taiwan University), Jih-Hsin Cheng (Institute of Mathematics, Academia Sinica), Qing-Ming Cheng (Fukuoka University), Shiu-Yuen Cheng (Hong Kong University of Science and Technology), Hung-Lin Chiu (National Central University), Qing Ding (Fudan University), Bo Guan (Ohio State University/ Xiamen University), Jiaxing Hong (Fudan University), Sen $\mathrm{Hu}$ (University of Science and Technology of China), Siu-Cheong Lau (Harvard University), Yng-Ing Lee (National Taiwan University), Xiangyu Liang (University of Warwick), Xiaochun Rong (Capital Normal University/ Rutgers University), Li-Sheng Tseng (University of California, Irvine), Mu-Tao Wang (Columbia University), Hongwei Xu (Zhejiang University), Mei-Lin Yau (National Central University), Entao Zhao (Zhejiang University), Xi-Ping Zhu (Sun Yat-Sen University)

\section{Topology, Geometry and Mathematical Physics}

Miranda C. N. Cheng (Institut de Mathématiques de Jussieu, Paris Diderot), Hai-Bao Duan (Academy of
Mathematics and Systems Science, Chinese Academy of Sciences), Fei Han (National University of Singapore), Lizhen Ji (University of Michigan), Naichung Conan Leung (The Chinese University of Hong Kong), Si Li (Boston University), Tao Li (Boston College), Chiu-Chu Melissa Liu (Columbia University), Kefeng Liu (Zhejiang University/ University of California, Los Angeles), Zhi Lu (Fudan University), Feng Luo (Rutgers University), Yi Ni (California Institute of Technology), Ruifeng Qiu (East China Normal University), Ser Peow Tan (National University of Singapore), Junwu Tu (University of Oregon), Loring W. Tu (Tufts University), Hao Xu (Harvard University), Yisong Yang (Polytechnic Institute of New York University), Jian Zhou (Tsinghua University), Shengmao Zhu (Zhejiang University)

\section{Statistical Theory, Methods, Applications, Probability, Financial Mathematics}

Hock Peng Chan (National University of Singapore), Guan-Yu Chen (National Chiao Tung University), Louis H. Y. Chen (National University of Singapore), Tailen Hsing (University of Michigan), Hsien-Kuei Hwang (Academia Sinica), Ching-Kang Ing (Academia Sinica), Wai Keung Li (The University of Hong Kong), Xiaotong Shen (University of Minnesota), Shuenn-Jyi Sheu (National Central University), Hansheng Wang (Peking University), Jane-Ling Wang (University of California, Davis), Naisyin Wang (University of Michigan), Xiaoqun Wang (Tsinghua University), Jun Yin (University of Wisconsin-Madison), Dapeng Zhan (Michigan State University)

\section{Dynamical System, Control Theory, Optimization, Functional Analysis, Fractals}

Kuo-Chang Chen (National Tsing Hua University), Chong-Qing Cheng (Nanjing University), Lixin Cheng (Xiamen University), Ai-Hua Fan (Université de Picardie Jules Verne), De-Jun Feng (The Chinese University of Hong Kong), Cheng-Hsiung Hsu (National Central University), Jiaxin Hu (Tsinghua University), Yunping Jiang (The City University of New York), Hanfeng Li (Chongqing University/ SUNY at Buffalo), Wan-Tong Li (Lanzhou University), Y. Charles Li (University of Missouri), Xiangyu Liang (University of Warwick), Song-Sun Lin (National Chiao Tung University), Yong Lin (Renmin University of China), Yiming Long (Nankai University), Yuefei Wang (Academy of Mathematics and Systems Science, Chinese Academy of Sciences), Zhiren Wang (Yale University), Zhiying Wen (Tsinghua University), Yuesheng Xu (Sun Yat-Sen University), Gaofei Zhang (Nanjing University), Guang Yuan Zhang (Tsinghua University)

\section{Computational Mathematics, Imaging, and Mathematical Biology}

Wei Cai (University of North Carolina at Charlotte), Raymond Hon-Fu Chan (The Chinese University of Hong Kong), Tony F. Chan (The Hong Kong University of Science 
and Technology), Falai Chen (University of Science and Technology of China), Li-Tien Cheng (University of California, San Diego), I-Liang Chern (National Chiao Tung University), Xianfeng Gu (State University of New York at Stony Brook), Shi Jin (Shanghai Jiao Tong University, China/ University of Wisconsin-Madison, USA), Chiu-Yen Kao (Claremont Mckenna College), Ming-Chih Lai (National Chiao Tung University), Ka Sing Lau (The Chinese University of Hong Kong), Fengyan Li (Rensselaer Polytechnic Institute), Michael Kwok-Po Ng (Hong Kong Baptist University), Qing Nie (University of California, Irvine), Weixiao Shen (National University of Singapore), Tony Wen-Hann Sheu (National Taiwan University), Huazhong Tang (Peking University, P.R. China), Wei-Cheng Wang (National Tsing Hua University), Weichung Wang (National Taiwan University), Xiao-Ping Wang (Hong Kong University of Science and Technology), Jack Xin (University of California, Irvine), Yan Xu (University of Science and Technology of China), Yongtao Zhang (University of Notre Dame), Hongkai Zhao (University of California, Irvine), Hao-Min Zhou (Georgia Institute of Technology)

\section{Partial Differential Equation}

Chiun-Chuan Chen (National Taiwan University), Shouxin Chen (Henan University), Jong-Shenq Guo (Tamkang University), Sze-Bi Hsu (National Tsing-Hua University), Zhen Lei (Fudan University), Dongsheng Li (Xi'an Jiaotong University), Tao Luo (Mathematical Sciences Center, Tsinghua University), Shuangjie Peng (Central China Normal University), Jenn-Nan Wang (National Taiwan University), Juncheng Wei (The Chinese University of Hong Kong/ University of British Columbia), Shusen Yan (University of New England, Australia), Tong Yang (City University of Hong Kong), Lei Zhang (University of Florida), Ping Zhang (Academy of Mathematics and System Sciences, Chinese Academy of Sciences), Wenming Zou (Tsinghua University) 